\title{
Event generators for heavy-ion physics, recent developments
}

\section{Gösta Gustafson}

Dept. of Astronomy and Theoretical Physics, Lund University

E-mail: gosta.gustafsonethep.lu.se

\begin{abstract}
A short review is presented for the most commonly used general purpose event generators for heavy ion physics, HIJING, AMPT, and EPOS. A new model, called Angantyr, is presented. It is (similar to HIJING) an extrapolation of pp dynamics to collisions with nuclei. Diffractive excitation of individual nucleons has significant effects in nucleus collisions, and here Angantyr is the first model which accounts for nucleon excitation in both projectile and target nuclei. General features in $\mathrm{p} A$ and $A A$ collisions are well reproduced. Future studies aim to investigate effects of fluctuations in more detail, and to also include effects of a high string density on collective effects, like high strangeness production and transverse flow.
\end{abstract}

Sixth Annual Conference on Large Hadron Collider Physics (LHCP2018)

4-9 June 2018

Bologna, Italy 


\section{Introduction}

The most important question in heavy ion scattering is: is a thermalized QGP plasma formed in heavy ion collisions? To answer this question some expected signals are higher rate for strange particles, lower rate for heavy bound states like $\psi$, and transverse flow giving higher $p_{\perp}$ and a same side ridge. A problem is here that many of these signals are seen also in high multiplicity pp events. Does this imply that a plasma is formed in pp collisions, or, alternatively, can the expected signals be the result of high matter density, without the formation of a thermalized state?

For pp collisions we have a number of highly successful general purpose event generators, based on multiple parton subcollisions followed by string or cluster hadronization. They work well all the way from soft to very hard scales.

For heavy ion collisions Monte Carlo generators are often aimed for specific features, expected in a plasma. In this talk I will concentrate on three commonly used general purpose event generators and one new. HIJING [1] and the new Angantyr model [2] are both extrapolating pp dynamics to $\mathrm{p} A$ and $A A$ collisions. Differences with respect to the data should here indicate effects of collectivity. In contrast AMPT [3] and EPOS [4] are both assuming the formation of a hot thermalized medium.

\section{Extrapolation of pp dynamics}

\subsection{The Glauber model}

The Glauber model [5] is the basis for many Monte Carlos for calculating cross sections and the number of interacting nucleons and binary $N N$ collisions. Rescattering, which in transverse momentum space is given by a convolution, is in impact parameter space simply given by a product. This implies that in a $\mathrm{p} A$ collision with target nucleon positions $\mathbf{b}_{v}(v=1, \ldots, A)$, the $S$-matrix factorizes:

$$
S^{(p A)}(\mathbf{b})=\prod_{v=1}^{A} S^{(p p, v)}\left(\mathbf{b}-\mathbf{b}_{v}\right)
$$

In high energy pp collisions the real part of the amplitude $A=i(1-S)$ is small, which indicates that the interaction is driven by absorption. If $\operatorname{Re}(A)$ can be neglected, $\left(S^{p p}\right)^{2}$ is the probability for no absorption. If we define the real "amplitude" $T(b) \equiv 1-S(b)$, the inelastic cross section is given by

$$
d \sigma_{\text {inel }} / d^{2} b=1-S^{2}=2 T-T^{2} .
$$

As $S$ factorizes, so does $S^{2}$. For $\mathrm{p} A$ collissions we therefore get

$$
d \sigma_{\text {inel }}^{p A} / d^{2} b=1-\prod_{v}\left(S^{p p, v}\right)^{2}
$$

\subsection{The wounded nucleon and the Fritiof model}

Białas, Bleszyński, and Czyż [6] noticed that the central particle density in $\mathrm{p} A$ collisions was proportional to the number of "wounded" (or "participant") nucleons, $N_{w}=1+N_{w}^{t}$ :

$$
\frac{d N^{p A}}{d \eta} \approx \frac{1+N_{w}^{t}}{2} \frac{d N^{p p}}{d \eta} .
$$


This feature was used in the early Fritiof model [7]. It was here assumed that two colliding nucleons are excited by longitudinal momentum exchange to masses $M_{A}$ and $M_{B}$, with distributions $\propto d M_{i}^{2} / M_{i}^{2}(i=A, B)$. They were then hadronized like strings, which on average give triangular particle distributions in rapidity. Similar distributions from wounded nucleons were later observed by Białas and Czyż in an analysis of dAu collisions [8]. At fixed target energies Fritiof actually worked better than expected (leaving little room for effects of a thermalized plasma), but with increasing energies hard parton sub-collisions become more and more important, and have to be properly included in a realistic model for nucleon-nucleon interaction.

\section{Commonly used general purpose MCs}

HIJING [1] is a MC which extrapolates pp interaction, with the aim that differences to data indicate effects of collective behaviour. Soft interaction is based on the Fritiof idea, but with excitation to higher energies, like in the DPM model, and soft radiation is included using the Ariadne dipole cascade [9] for $p_{\perp}$ below a cut $p_{0}$. Hard multi-parton interactions are added à la PYTHIA for $p_{\perp}$ above $p_{0}$, using nuclear pdf:s suppressed $c f$. to $A$ proton pdf:s. A new version in $\mathrm{C}++$, called HIJING++, is recently presented [10].

AMPT [3] assumes that a hot dense medium is formed. It uses HIJING for initial conditions, and contains four components: (i) initial conditions from the parton state in HIJING before its hadronization, (ii) expansion in a partonic cascade until freezeout, (iii) the partons are connected to strings and hadronized using PYTHIA, (iv) the hadron soup expands in a hadronic cascade till the density is low enough.

EPOS [4] separates the collision in two components: a high density "core" and a low density "corona". There is no explicit Glauber calculation. Instead partonic sub-collisions are calculated via Gribov-Regge theory, with elementary scatterings represented by cut pomerons or "parton ladders". These partons form strings, which break into segments by $q \bar{q}$ formation. Within the core, gluons within a bin in $\eta$ form a cluster which expands until freezeout, while in the corona they fragment directly to hadrons. In the recent version, EPOS-LHC [4], the hydro expansion is paramerized instead of generated from hydrodynamics, as in earlier versions. This also implies that the time for one $\mathrm{PbPb}$ collision is reduced from 1 hour to tenths of a second.

\section{The rôle of fluctuations}

As early pointed out by Gribov [11], a proton can be diffractively excited after the first (or a subsequent) interaction, which has a significant effect on the result. In impact parameter space (used in the Glauber model) diffraction is most easily treated in the Good-Walker formalism. Here diffractive excitation is described as the result of fluctuations in the proton substructure. A proton is a coherent mixture of parton cascades, where cascades with many partons have a larger cross section than cascades with fewer partons. The non-absorbed wave after a collision is therefore not a pure proton state, but a mixture of a proton and excited states with higher masses. The cross section for diffractive excitation is then given by the fluctuations in the amplitude:

$$
d \sigma_{\text {diffex }} / d^{2} b=\left\langle T^{2}\right\rangle-\langle T\rangle^{2} .
$$


When both projectile and target are fluctuating, as in pp collisions, single excitation of the projectile, of the target, and double diffraction are given by different averages over projectile and target states, see e.g. ref. [12]. An important point in $A A$ collisions is then that a projectile (target) nucleon stays in the same diffractive eigenstate (the same partonic cascade) throughout the passage through a target (projectile) nucleus. This implies that in collisions with nuclei, averages of higher moments of $T$ are involved, not only the first and second moments as in eq. (4.1).

In Fritiof or the wounded nucleon model, the mass distribution for an absorbed nucleon is given by $d P / d M^{2} \sim 1 / M^{2}$. For a diffractively excited nucleon we also have $d P / d M_{X}^{2} \sim 1 / M_{X}^{2}$. Thus we can talk about two different types of wounded nucleons, which give very similar contributions to the final state.

Strikman and coworkers have in several papers since 1991 studied effects of fluctuations in $\mathrm{p} A$ collisions, see ref. [13] and further references in there. This formalism, called GG or GGCF, accounts for fluctuations in the projectile, but averages over target nucleon states. This gives a correct result provided absorptively wounded and diffractively wounded nucleons give the same contribution to the final state [12]. In $A A$ collisions it is, however, important to take into account nucleon fluctuations in both the projectile and the target nuclei.

\section{A new model: Angantyr}

Angantyr is a new model for high energy $\mathrm{p} A$ and $A A$ collisions [2]. To my knowledge it is the only model for $A A$ collisions, which accounts for fluctuations in both projectile and target nucleons. It is an extrapolation from pp interaction with a minimum of free parameters, inspired by the Fritiof model and with pp interaction from PyтнiA8. The MC is available in the PYTHiA8 package.

The structure of a final state is illustrated in fig. 1 left panel, which shows a $\mathrm{p} A$ interaction with four wounded nucleons. Two nucleons (labeled $p$ and $N_{1}$ ) are connected by a gluon chain all the way from projectile to target. A third nucleon $\left(N_{2}\right)$ is, via a shorter gluon chain, connected to the first chain at some intermediate point. Finally the fourth nucleon $\left(N_{3}\right)$ is diffractively wounded. Each of the gluon chains should here be interpreted as including multiple parton interactions, in accordance with the model implemented in PYTHIA8.

Fig. 1 right panel shows the average number of participating nucleons $v s$ centrality (determined from forward detectors) in $\mathrm{pPb}$ collisions. The MC results are compared with data from ATLAS [14]. Including fluctuations in the Glauber analysis (going from "plain Glauber" to GGCF with $\Omega=0.11$ and $\Omega=0.20$ ) increases the number of participants for low centrality. In Angantyr, which includes even more fluctuations, this increase is even higher. We also note that if centrality is determined by the impact parameter, $b$, instead of by forward activity (the dashed red line), the result is almost constant for centralities below 10\%. This means that the increase for small centralities is a forward-central correlation, rather than a correlation with impact parameter.

Fig. 2 shows results for XeXe collisions from ALICE [15], compared with results from a set of MC generators. The left figure shows $d N_{c h} / d \eta$ for 0-5\% central events. We note that Angantyr agrees with data to within 5-10\%. The right figure shows $\frac{2}{\left\langle N_{\text {part }}\right\rangle} d N_{c h} / d \eta v s\left\langle N_{\text {part }}\right\rangle$. We note here in particular that Angantyr (the dotted red line) reproduces the rise for high multiplicities better than the other MCs. In future work we aim to investigate closer if this is an effect of the larger fluctuations in Angantyr, or if it is due to some other effect. 

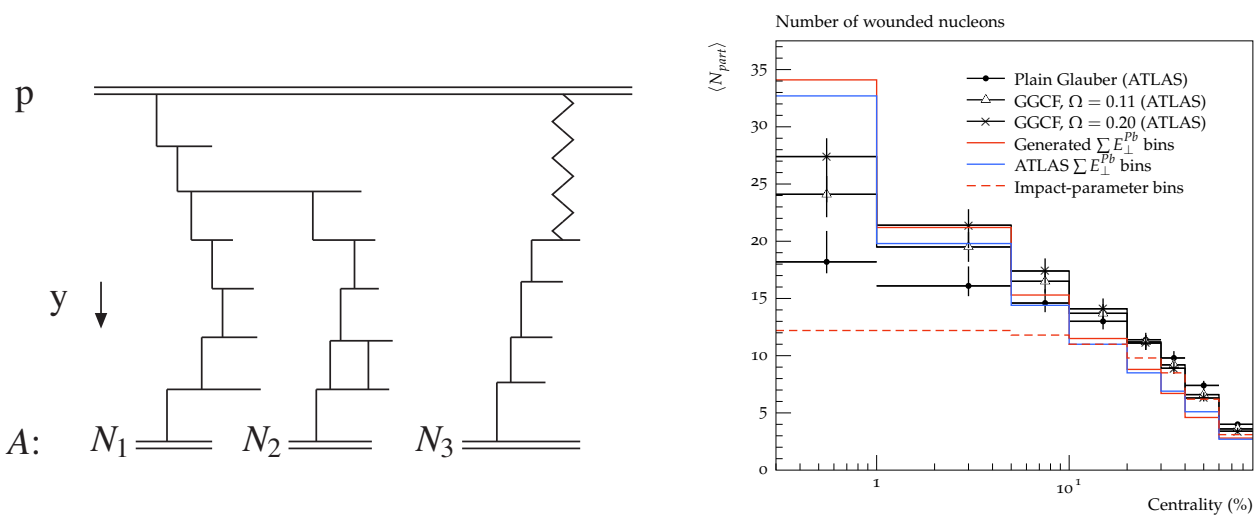

Figure 1: Left plot: A pA interaction with four wounded nucleons. The thin lines represent gluons, and the zigzag line a pomeron. Right plot: Average number of participating nucleons $v s$ centrality (determined from forward detectors) in $\mathrm{pPb}$ collisions at 5.02 TeV. Data from ATLAS [14] with results for plain Glauber and the GGCF model with widths determined by $\Omega=0.11$ and by $\Omega=0.20$. The results from Angantyr are obtained with centrality determined by $\sum E_{\perp}^{P b}$ from the MC (red line), from ATLAS data (blue line), or determined by the impact parameter $b$ (dashed red line).
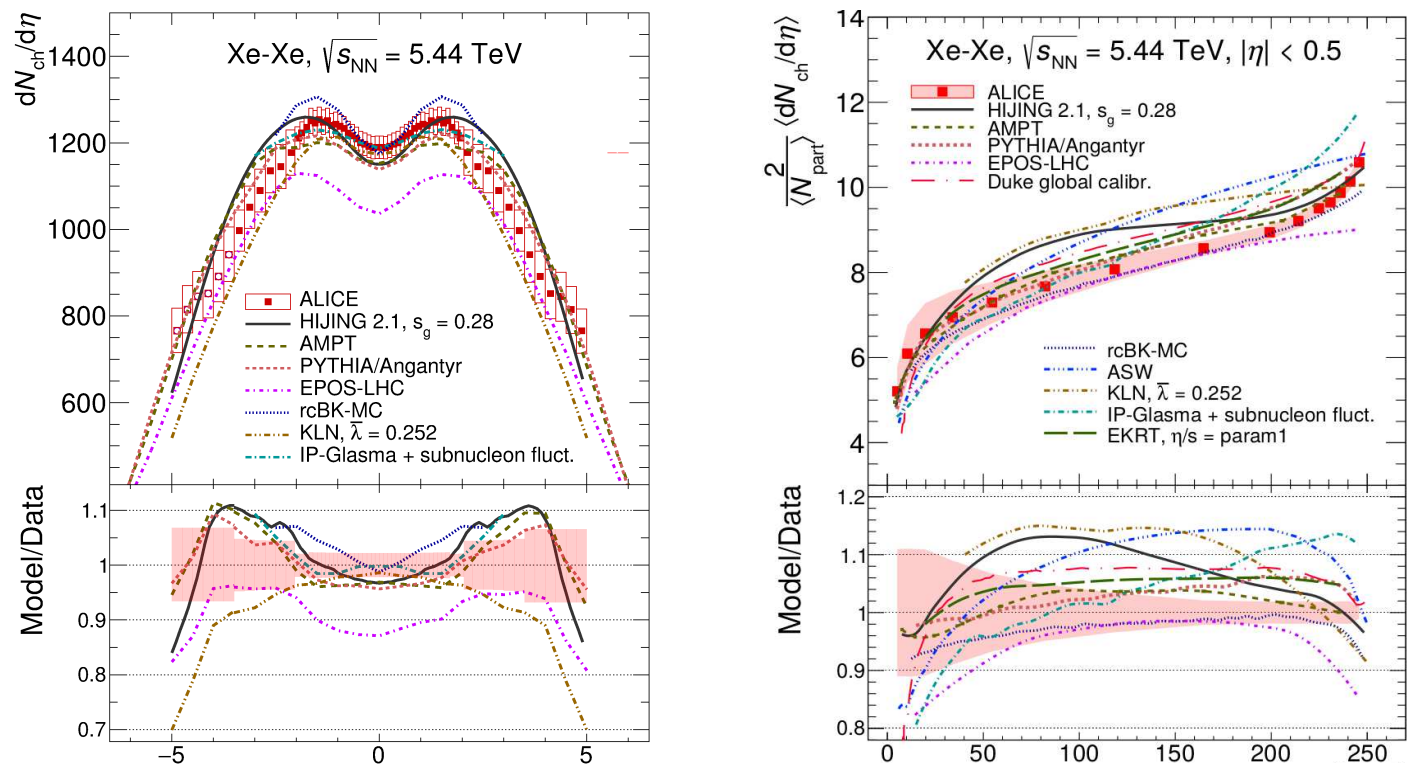

Figure 2: Results for XeXe collisions at 5.44 TeV from ALICE [15], compared with results from a set of MC generators. Left plot: $d N_{c h} / d \eta v s \eta$ for $0-5 \%$ central events. Right plot: $\frac{2}{\left\langle N_{\text {part }}\right\rangle} d N_{c h} / d \eta v s\left\langle N_{\text {part }}\right\rangle$.

\section{Outlook}

Angantyr reproduces general hadron distributions. It is special as it includes diffraction and fluctuations in both projectile and target nucleons. Fluctuations are seen to be particularly important for estimates of $N_{\text {part }}$, while other effects need more study. The next step is to include flow-like effects seen in pp, in the extrapolation to collisions with nuclei. The high string density in high energy pp collisions ought to give high energy density, resulting in more strangness [16], as well 
as a transverse flow [17]. We are presently working on including these effects into Angantyr.

\section{References}

[1] X.-N. Wang and M. Gyulassy, HIJING: A Monte Carlo model for multiple jet production in pp, pA and AA collisions, Phys. Rev. D44 (1991) 3501.

[2] C. Bierlich, G. Gustafson, L. Lönnblad, and H, Shah, The Angantyr model for Heavy-Ion Collisions in PYTHIA8, arXiv:1806.10820.

[3] Z.-W. Lin, C. M. Ko, B.-A. Li, B. Zhang, and S. Pal, A Multi-phase transport model for relativistic heavy ion collisions, Phys. Rev. C72 (2005) 064901 [nucl-th/ 0411110 ].

[4] T. Pierog et al., EPOS LHC: Test of collective hadronization with data measured at the CERN Large Hadron Collider, Phys. Rev. C92 (2015) 034906 [arXiv: 1306.0121 ].

[5] R. J. Glauber, Cross-sections in deuterium at high-energies, Phys. Rev. 100 (1955) 242.

[6] A. Bialas, M. Bleszynski, and W. Czyz, Multiplicity Distributions in Nucleus-Nucleus Collisions at High-Energies, Nucl. Phys. B111 (1976) 461.

[7] B. Andersson, G. Gustafson, and B. Nilsson-Almqvist, A Model for Low p(t) Hadronic Reactions, with Generalizations to Hadron-Nucleus and Nucleus-Nucleus Collisions, Nucl. Phys. B281 (1987) 289.

[8] A. Bialas and W. Czyz, Wounded nucleon model and Deuteron-Gold collisions at RHIC, Acta Phys. Polon. B36 (2005) 905.

[9] L. Lönnblad, ARIADNE version 4: A Program for simulation of QCD cascades implementing the color dipole model, Comput. Phys. Commun., 71 (1992) 15.

[10] G. Papp et al. First Results with HIJING++ on High-energy Heavy Ion Collisions, (2018), arXiv:1805.02635.

[11] V.N. Gribov, Glauber corrections and the interaction between high-energy hadrons and nuclei, Sov. Phys. JETP, 29 (1969) 483.

[12] C. Bierlich, G. Gustafson, and L. Lönnblad, Diffractive and non-diffractive wounded nucleons and final states in pA collisions, JHEP 10 (2016) 139 [arXiv: 1607.04434 ].

[13] M. Alvioli, L. Frankfurt, V. Guzey, and M. Strikman, Revealing "flickering" of the interaction strength in pA collisions at the CERN LHC, Phys. Rev. C90 (2014) 034914 [arXiv: 1402 . 2868].

[14] G. Aad et al., Measurement of the centrality dependence of the charged-particle pseudorapidity distribution in proton-lead collisions at $\sqrt{s_{\mathrm{NN}}}=5.02 \mathrm{TeV}$ with the ATLAS detector, Eur. Phys. J. C76 (2016) 199 [arXiv:1508.00848].

[15] S. Acharya et al., Centrality and pseudorapidity dependence of the charged-particle multiplicity density in Xe-Xe collisions at $\sqrt{s_{\mathrm{NN}}}=5.44 \mathrm{TeV}$, arXiv:1805.04432.

[16] C. Bierlich, G. Gustafson, L. Lönnblad, and A. Tarasov, Effects of Overlapping Strings in pp Collisions, JHEP 03 (2015) 148 [arXiv: 1412 . 6259].

[17] C. Bierlich, G. Gustafson, and L. Lönnblad, Collectivity without plasma in hadronic collisions, Phys. Lett. B779 (2018) 58 [arXiv: 1710 . 09725]. 